\section{Vol No: 4, Issue: 2}

Received Date: Jun 13, 2019

Published Date: Jul 2, 2019

\section{Yan Leyfman}

Penn State College of Medicine, Hershey, USA.

\section{Corresponding Author:}

\section{Yan Leyfman*}

Penn State College of Medicine, Hershey, Pennsylvania, USA.

\title{
Confabulations in a Chronic Alcoholic: A Subtle Presentation of Korsakoff Syndrome
}

\section{ABSTRACT:}

Wernicke Encephalopathy is an acute neuropsychiatric condition, characterized by confusion, nystagmus, ataxia, and ophthalmoplegia, resulting from thiamine (Vitamin B1) deficiency, typically secondary to alcohol abuse. Failure to properly diagnose, which occurs in $80 \%$ of the time, can result in gradual progression to irreversible Korsakoff Syndrome, characterized by irreversible personality changes, and anterograde and retrograde amnesia. The present case report seeks to highlight this patient's chronology to Korsakoff Syndrome and our attempted interventions.

Keywords: Wernicke's Encephalopathy; Korsakoff's Syndrome; Delirium; Confusion; Thiamine Deficiency; Alcohol Abuse.

\section{CASE REPORT}

Benign A previously healthy 73-yearold male with a 10-year history of alcohol abuse presented to the ED with confusion, lethargy and weakness for 2 days and 2 episodes of vomiting in the past week. Over the past year, he has been eating less, acting more reserved, and over the past 2 months exhibited sharp mental decline, including shortterm memory loss and personality changes. On admission, he was afebrile with HR 100 bpm, BP 136/97 mmHg, RR $18 \mathrm{bpm}$, and $\mathrm{O} 2$ sat $95 \%$ on a 2L nonrebreather mask. The patient appeared cachectic and somnolent. His skin was warm, lungs were clear to auscultation and heart in regular rate and rhythm without murmurs. On neurological examination, the patient was nonverbal and oriented to only name. Labs showed an elevated ammonia level of $25 \mu \mathrm{mol} / \mathrm{L}$, procalcitonin of $0.37 \mathrm{ng} / \mathrm{mL}$, lactic acid level of $5.0 \mathrm{mmol} / \mathrm{L}$ with an anion gap of 23 , and a negative urine toxicity screen.
The EKG showed normal sinus rhythm with rate of $82 \mathrm{bpm}$, with intermittent PVCs and PR interval of $180 \mathrm{~ms}$, and QTC was prolonged at $511 \mathrm{~ms}$. The CT of the abdomen was negative. The patient exhibited a CIWA score of 11. MOCA score could not be performed due to the patient's obtunded state. Due to the patient's extensive drinking history, he was admitted for altered mental status and treated with lorazepam, potassium chloride, Vitamin B1, folic acid and IV fluids. In the subsequent days, memory loss and confabulations were noted clinching a diagnosis of Korsakoff Syndrome. The patient was eventually discharged to rehabilitation.

\section{DISCUSSION}

In the USA, $40 \%$ of hospitalizations are attributed to alcoholism [1]. Chronic Alcoholism may present with a classic triad of confusion, oculomotor dysfunction and gait ataxia consistent with Wernicke encephalopathy, an 
acute but reversible syndrome due to thiamine deficiency where the most common symptoms in order of prevalence include mental status changes, ocular signs and gait disturbance [2,3]. However, persistent thiamine deficiency, especially in alcoholics, can progress to Korsakoff Syndrome, an irreversible disorder characterized by confabulations, disorientation, irreversible personality changes, and anterograde and retrograde amnesia [4]. Although both are largely clinical diagnoses, brain MRI can be performed in ambiguous cases showing low-density lesions in the periventricular structures, diencephalon, and midbrain with atrophy of the mamillary bodies, cerebellum and cerebrum $[5,6]$. However, imaging will not affect course of treatment. Although both conditions are treated with alcoholic abstinence and thiamine supplementation (short-term high dose IV Vitamin B1 followed by lower dose in Wernicke and long-term PO supplementation in Korsakoff), Korsakoff Syndrome is additionally managed with rehabilitation, psychiatric therapy and memory strengthening exercises due to its irreversibility $[7,8]$.

This case demonstrates sequalae of chronic alcoholism, initially diagnosed and treated as Wernicke encephalopathy but later amended to Wernicke-Korsakoff due to confabulations, personality changes and memory loss, as the patient became more aroused. It is likely that chronic alcoholism, poor diet, and lack of medical care contributed to the patient's present state. In summary, it is critical to consider Wernicke-Korsakoff Syndrome in the differential for altered mental status, especially in chronic alcoholics.

\section{REFERENCES}

1. Maldonado JR, Sher Y, Ashouri JF, Hills-Evans $\mathrm{K}$, et al. (2014). The "Prediction of Alcohol Withdrawal Severity Scale" (PAWSS): systematic literature review and pilot study of a new scale for the prediction of complicated alcohol withdrawal syndrome. Alcohol. 48(4): 375-390.

2. Vasan S, Kumar A. (2018). Wernicke Encephalopathy. StatPearls Publishing, Florida, USA.

3. Flynn A, Macaluso M, D'Empaire I and Troutman MM. (2015). Wernicke's Encephalopathy: Increasing Clinician Awareness of This Serious, Enigmatic, Yet Treatable Disease. Prim Care Companion CNS Disord. 17(3): DOI: 10.4088/PCC.14r01738
4. Gossman WG and Newton EJ. (2018). Wernicke-Korsakoff Syndrome. StatPearls Publishing, Florida, USA.

5. Jung YC, Chanraud S and Sullivan EV.(2012). Neuroimaging of Wernicke's encephalopathy and Korsakoff's syndrome. Neuropsychol Rev. 22(2): 170-180.

6. Sullivan EV and Pfefferbaum A. (2009). Neuroimaging of the Wernicke-Korsakoff Syndrome. Alcohol Alcohol. 44(2): 155-165.

7. Charness ME, Aminoff MJ and Wilterd JL. (2018). Overview of the Chronic Neurologic Complications of Alcohol. UpToDate, Massachusetts, USA.

8. Kopelman MD, Thomson AD, Guerrini I and Marshall EJ. (2009). The Korsakoff Syndrome: Clinical Aspects, Psychology and Treatment. Alcohol Alcohol. 44(2): 148154. 